“C 2016 IEEE. Personal use of this material is permitted. Permission from IEEE must be obtained for all other uses, in any current or future media, including reprinting/republishing this material for advertising or promotional purposes, creating new collective works, for resale or redistribution to servers or lists, or reuse of any copyrighted component of this work in other works." 


\title{
Multilevel Robust Design Optimization of a Superconducting Magnetic Energy Storage Based on a Benchmark Study
}

\author{
Gang Lei, Jianguo Zhu, Chengcheng Liu, Mohammad Jafari, and Youguang Guo
}

\begin{abstract}
Superconducting magnetic energy storage (SMES) systems with different superconducting materials are attracting great attentions and funding from the governments around the world because they are promising large-scale energy storage devices for future smart grid. Due to the high cost of SMES, its manufacturing quality and operation reliability have to be investigated in the design optimization stage. This paper presents a robust design optimization framework to solve this issue based on a benchmark problem, TEAM problem 22. The proposed robust design optimization is based on a technique called design for Six-Sigma. Meanwhile, a modified multilevel optimization strategy is employed to reduce the computation cost of finite element analysis due to high-dimensional design space and Monte Carlo analysis. As shown, the reliability and manufacturing quality of the investigated SMES after robust optimization have been increased greatly.
\end{abstract}

Index Terms-Manufacturing quality, multilevel optimization, robust optimization, superconducting magnetic energy storage.

\section{INTRODUCTION}

$\mathrm{W}$ ITH the development of smart grid, more and more distributed energy sources, such as wind, solar and other renewable energy power, will be connected to the main grid via a number of microgrid systems. However, due to the uncertainties of these renewable energy power sources, how to maintain the grid reliability and power quality is a big challenge in power systems as large amount of power may be required instantaneously for many situations, for example, sudden disconnection of some energy sources from the main grid. In order to deal with this challenge, superconducting magnetic energy storage (SMES), as a kind of grid-enabling device, has been investigated by many researchers [1]-[4].

In general, there are two types of SMES systems in terms of superconducting temperature. The first one is the lowtemperature SMES, where the most widely used superconducting materials are $\mathrm{NbTi}$ and $\mathrm{Nb} 3 \mathrm{Sn}$ [5]. However, liquid helium is generally required as the coolant for this kind of SMES. Thus, its cost is usually very high and this limits the applications of this kind of SMES. The second type is the high-temperature superconductor (HTS) SMES. Some SMESs made by different types of HTSs, such as BSCCO and YBCO, have been actively designed, fabricated and tested

G. Lei, J. G. Zhu, C. C. Liu, M. Jafari, and Y. G. Guo are with School of Electrical, Mechanical and Mechatronic Systems, University of Technology, Sydney, NSW, 2007, Australia. (Corresponding author: G. Lei; email: Gang.Lei@uts.edu.au). experimentally, for example, in-grid operations, around the world [6]-[9]. Recently, a promising movable HTS SMES system has been developed to maintain a long-term stable operation of the power system [10].

A major challenge in SMES application at present is the very high cost, mainly including the material costs of superconducting coils and cooling units, for example, liquid helium. Therefore, manufacturing quality and operation reliability should be investigated in the design optimization of SMES, particularly the design of superconducting coils. The main aim of this consideration is to ensure that the theoretically designed SMES can achieve the expected performance in terms of unavoidable variations due to practical manufacturing tolerances and excitation fluctuations. This will be very beneficial to the industrial applications of SMES systems from the perspective of economic efficiency. This work will investigate this issue based on a highdimensional benchmark problem of SMES.

\section{DESIGN OPTIMIZATION OF SMES}

In the design of different types of SMES systems, the core technology is the design optimization of superconducting coil as it determines the system's performance. Solenoid and toroidal coils are the two main topologies for the superconducting coils. To obtain the best system performance, many parameters have to be investigated. In general, the following objectives, constraints and parameters can be investigated in the design optimization stage of a SMES.

Firstly, regarding the design optimization objectives, some popular ones are maximizing storage energy/energy density and coil inductance, and minimizing the coil volume, cost by kilogram and average stray field [4]-[9], [11], [12]. For HTS SMES, hoop stress is also an important objective due to the material property of HTSs. In this case, the mechanical stresses induced in the windings by electromagnetic force will result in instability in the superconducting coils. Therefore, multiphysics analysis of electromagnetic field and structural analyses should be developed for the corresponding design and optimization [4], [7].

Secondly, considering the optimization constraints, the most important one is related to the quenching condition of the superconducting material. This condition generally shows the relation of the current density and the maximum value of magnetic flux density in SMES [5], [11]-[15]. In addition, some optimization objectives, such as the volume and average 
stray field, can be easily converted into constraints by the given requirements.

Thirdly, in terms of design parameters, there are many options. Coil dimensions including inner and outer diameters and height are the popular ones for the core design if the coil topology is given [4], [5], [8], [11]-[15]. Then, some parameters from coil topology can be included in the optimization as well. For example, considering a type of single-pole double pancake coil (DPC) for SMES, the winding turns, DPC number and gap between DPC are also important parameters for optimization [7]. Moreover, current density is also a key optimization parameter as it is directly related to the storage energy and quenching condition [11]-[15].

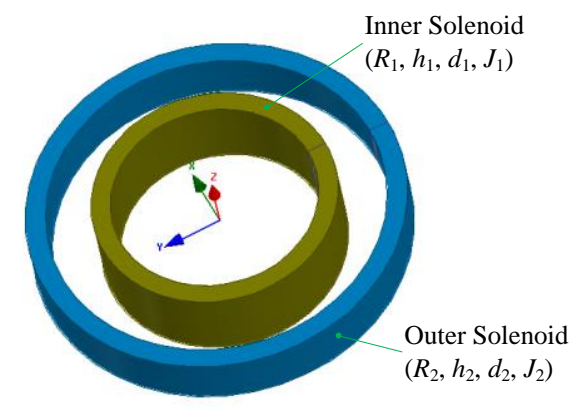

Fig. 1 Illustration of two solenoids in the studied SMES

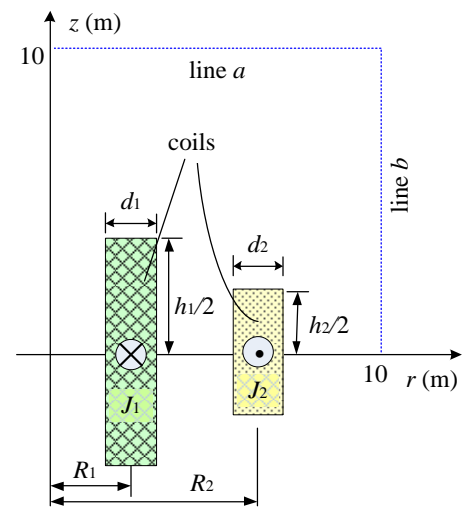

Fig. 2 The geometry configuration of SMES (axisymmetric)

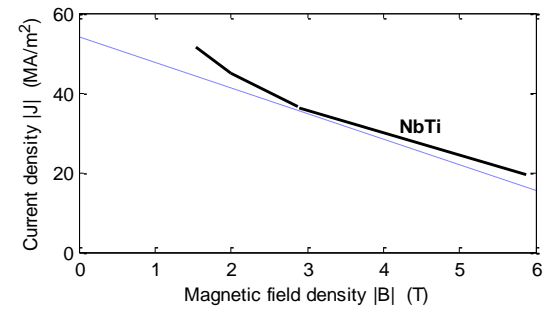

Fig. 3 Critical curve of superconducting material

Taking the TEAM benchmark problem 22 as an example, Fig. 1 shows the coil topology of this SMES. Due to the axisymmetric structure, only a 2D cross-section of this SMES as shown in Fig. 2 will be required for the design analysis and optimization. As shown, this SMES consists of two solenoids. There are totally eight parameters, where $\left(R_{1}, h_{1}, d_{1}\right)$ is the dimension of the inner solenoid, $J_{1}$ is the current density of the inner solenoid, $\left(R_{2}, h_{2}, d_{2}\right)$ and $J_{2}$ are the corresponding parameters of the outer solenoid. Table I tabulates the ranges of these parameters [16]-[18]. There are many forms of the optimization model for this benchmark problem. In this work, the optimization model is defined as

$$
\begin{gathered}
\text { Min }: f(\mathbf{x})=\sqrt{\frac{\sum_{i=1}^{21}\left|B_{\text {stray }}^{\mathrm{i}}\right|^{2}}{21 B_{\text {norm }}}} \\
\text { s.t. }: g_{1}(\mathbf{x})=\left|E / E_{\text {ref }}-1\right|-0.01 \leq 0 \\
g_{2}(\mathbf{x})=\left|B_{\text {max }}\right|-\min \left\{54-\left|J_{\mathrm{i}}\right|\right\} / 6.4 \leq 0
\end{gathered}
$$

where $B_{\text {stray }}^{\mathrm{i}}(i=1,2, \ldots, 21)$ is the stray field on 21 points with the same space along lines $a$ and $b$ as shown in Fig. 2, $B_{\text {norm }}$ a normalized factor with value $3 \mathrm{mT}, B_{\max }$ the maximum of magnetic field, $E$ and $E_{\text {ref }}(180 \mathrm{MJ})$ are the practical and expected energy storages of this SMES, respectively. $g_{2}$ is related to the quenching condition of the used superconducting material, NbTi. As shown in Fig. 3, the black bold curve is the true critical curve. In the optimization, its linear approximation illustrated as a blue dashed-line and expressed as $g_{2}$ in (1) will be used as the limit.

TABLE I

OPTIMIZATION PARAMETERS FOR SMES

\begin{tabular}{ccccc}
\hline \hline Var. & Unit & Min. & Max. & Tolerance \\
\hline$R_{1}$ & $\mathrm{~m}$ & 1.0 & 4.0 & 0.01 \\
$R_{2}$ & $\mathrm{~m}$ & 1.8 & 5.0 & 0.01 \\
$h_{1} / 2$ & $\mathrm{~m}$ & 0.1 & 1.8 & 0.01 \\
$h_{2} / 2$ & $\mathrm{~m}$ & 0.1 & 1.8 & 0.01 \\
$d_{1}$ & $\mathrm{~m}$ & 0.1 & 0.8 & 0.005 \\
$d_{2}$ & $\mathrm{~m}$ & 0.1 & 0.8 & 0.005 \\
$J_{1}$ & $\mathrm{MA} / \mathrm{m}^{2}$ & 10.0 & 30.0 & $1 \% * J_{1}$ \\
$J_{2}$ & $\mathrm{MA} / \mathrm{m}^{2}$ & 10.0 & 30.0 & $1 \% * J_{2}$ \\
\hline \hline
\end{tabular}

\section{RoBUST OPTIMIZATION MODEL}

From the perspective of industrial design, there are three kinds of models, deterministic, reliability and robust models. Model (1) is a deterministic model because all parameters in it are deterministic, meaning that the practical SMES coil parameters after manufacturing are same as those obtained from theoretical optimization. Obviously, this is not always true in engineering as there are many unavoidable variations in the manufacturing. Similarly, there are some variations in the operation of SMES, such as the applied voltage and excitation current. Therefore, there are some variations between the theoretically designed and practical SMES coils. From previous experience, it is found that there are many unavoidable manufacturing tolerances for the dimension and current fluctuations in the operation of this SMES. All these variations are listed in the Table I as well [13], [14]. Therefore, all parameters are actually variables rather than deterministic 
values in the optimization. Consequently, all objectives and constraints in model (1) are variables and should be expressed as probability functions, instead of deterministic values.

Following the idea aforementioned, the model (1) can be converted into a robust design model based on a technique called design for Six Sigma (DFSS). In this technique, the design parameters are assumed to follow normal distributions with different means $(\mu)$ and standard deviations $(\sigma)$, thus to reflect the manufacturing tolerances and operation variations. Consequently, the objectives and constraints should be in the form of functions characterized by means and stand deviations of the initial ones. From this prospective, the robust design model based on DFSS for model (2) can be defined as

$$
\begin{array}{ll}
\text { Min : } & \mu_{f}(\mathbf{x}) \\
\text { s.t.: } & \mu_{g_{\mathrm{i}}}(\mathbf{x})+n \sigma_{g_{\mathrm{i}}}(\mathbf{x}) \leq 0, i=1,2, \\
& \mathbf{x}_{1}+n \sigma_{\mathbf{x}} \leq \mu_{\mathbf{x}} \leq \mathbf{x}_{\mathbf{u}}-n \sigma_{\mathbf{x}}
\end{array}
$$

where $\mu_{f}, \mu_{g i}$ and $\mu_{\mathbf{x}}$ are means of objective, constraint and variables, respectively, $\sigma_{f}, \sigma_{g i}$ and $\sigma_{\mathbf{x}}$ their standard deviations, $\mathbf{x}_{l}$ and $\mathbf{x}_{u}$ the lower and upper boundaries of $\mathbf{x}$, respectively, and $n$ is the sigma level. Generally, $n$ can be easily transformed to a probability value/ reliability in terms of a standard normal distribution. To achieve the highest profit, $6 \sigma$ level $(n=6)$ has been adopted in many companies worldwide nowadays as its equivalent defects per million opportunities (DPMO) is only 3.4 from the perspective of long-term quality control and management. As a comparison, $3 \sigma$ sigma level $(n=3)$ is actually equivalent to 66,803 DPMO, which is obviously insufficient from the perspective of quality control [19]-[22]. Meanwhile, to estimate the $\mu$ and $\sigma$ in (2), Monte Carlo analysis (MCA) is usually required. MCA is a classic statistical analysis technique for characterizing the uncertainty based on repeated random sampling. The sample size is usually big, for example, 10,000. In the implementation, we first generate 10,000 random samples for the design parameter $\mathbf{x}$ by using standard normal distribution, then 10,000 options of the design schemes can be achieved, resulting in 10,000 solutions of the objectives and constraints. Based on them, the means and standard deviations of them can be evaluated.

\section{Multilevel Optimization Method}

As finite element model (FEM) is employed to analyze the field distribution of the SMES in the optimization, the computation costs of optimization (1) and (2) are always huge due to the high dimension. For example, about $8 * 5 * 200=$ 8,000 FEM points are required if genetic algorithm (GA) is used to optimized the FEM of this SMES, where 8 is the dimension, $8 * 5$ is the population size of GA in each iteration, 200 is the average iteration numbers to obtain the optimization results. In addition, for model (2), the computation cost of MCA used to estimate $\mu$ and $\sigma$ is huge due to the big sample. Therefore, efficient optimization strategy/method is required.

To solve this issue, a method called multi-level optimization method presented for electrical drive systems in our previous work will be introduced in this work. The multilevel optimization method has two main implementation steps. Firstly, divide the initial high dimensional design space into two low-dimensional subspaces in terms of the significance order of all parameters, and call them the significant factors subspace (X1) and non-significant factors space (X2). In this step, sensitivity analysis techniques are required, such as local sensitivity analysis (LSA) and design of experiment. Secondly, optimize these three subspaces sequentially till convergence criterion is met. In addition, an approximation model, Kriging model will be used as surrogate models of FEM to reduce the computation cost. [23], [24].

Regarding the SMES studied in this work, Table II lists the analysis data of LSA. As shown, a two-subspace structure, $\mathrm{X} 1=\left\{R_{2}, h_{2}, d_{2}, J_{2}\right\}$ and $\mathrm{X} 2=\left\{R_{1}, h_{1}, d_{1}, J_{1}\right\}$ can be applied to the multilevel optimization. However, it is found that the obtained approximation model has insufficient accuracy due to the highly nonlinearity of dimension and excitation parameters. Therefore, it is better to separate them. And finally, a threelevel structure is required for the optimization of this SMES, where $\mathrm{X} 1=\left\{R_{2}, h_{2}, d_{2},\right\}, \mathrm{X} 2=\left\{J_{1}, J_{2}\right\}$ and $\mathrm{X} 3=\left\{R_{1}, h_{1}, d_{1}\right\}$. As each subspace has up to three parameters, sequential optimization method (SOM) will be employed to further improve the optimization efficiency. SOM is an efficient optimization strategy for low-dimensional electromagnetic design problem. The required FEM computation cost of FEM is around $1 / 10$ of the traditional method, for example, optimizing FEM by GA [15], [20]. These are the two modifications of the multilevel optimization method used in this work. Finally, it should be noted that this multilevel optimization method can be applied for both deterministic optimization model (1) and robust optimization model (2).

\section{OPTIMIZATION RESULTS AND DISCUSSIONS}

Table III lists the optimization results for three optimization methods. The first one is the single level optimization method based on deterministic approach (DA) model (2). It optimizes all eight parameters at one level. The second one is the multilevel optimization method based on (2). The last one is the multilevel optimization based on robust approach (RA) model (3). Tables IV and $\mathrm{V}$ list the results of SMES performance and reliability after MCA for the multilevel optimal solutions given in Table III. Through comparison, the following conclusions can be drawn.

(1) Multilevel optimization methods can present better optimization results compared with single level optimization, such as higher energy and lower mean stray filed. The multilevel optimization based on DA model has the best performance among them, such as smallest stray field $0.98 \mathrm{mT}$ and objective value 0.331 .

(2) Computation cost of FEM required by multilevel RA optimization is only $15.28 \%(721 / 4720)$ of that of single level optimization method.

(3) The probability of failure (POF) for batch production in industry (for example, 10,000 products) of DA optimum is around $2.38 \%$, which is larger than that of RA one (almost 0 ) as shown in Table V. 
TABLE II

SENSITIVITY ANALYSIS FOR LSA

\begin{tabular}{lllllll}
\hline \hline \multirow{8}{*}{ Par. } & \multicolumn{7}{l}{ Amplitude variations of parameter $(\delta)$} & & Sensitivity \\
& $-20 \%$ & $-10 \%$ & 0 & $10 \%$ & $20 \%$ & \\
\hline$R_{1}$ & 0.3460 & 0.1755 & 0 & -0.1756 & -0.3460 & 0.2608 \\
$R_{2}$ & -0.7293 & -0.4497 & 0 & 0.6690 & 1.6125 & 0.8651 \\
$h_{1} / 2$ & 0.1936 & 0.0947 & 0 & -0.0913 & -0.1787 & 0.1396 \\
$h_{2} / 2$ & -0.4840 & -0.2636 & 0 & 0.3076 & 0.6717 & 0.4317 \\
$d_{1}$ & 0.1937 & 0.0951 & 0 & -0.0914 & -0.1792 & 0.1399 \\
$d_{2}$ & -0.4780 & -0.2597 & 0 & 0.3015 & 0.6672 & 0.4266 \\
$J_{1}$ & 0.1929 & 0.0946 & 0 & -0.0910 & -0.1782 & 0.1392 \\
$J_{2}$ & -0.4765 & -0.2587 & 0 & 0.3012 & 0.6662 & 0.4256 \\
\hline \hline
\end{tabular}

TABLE III

OPTIMIZATION RESULTS FOR SMES

\begin{tabular}{lllll}
\hline \hline Par. & \multirow{2}{*}{ Unit } & $\begin{array}{l}\text { Single level } \\
\text { DA }\end{array}$ & $\begin{array}{l}\text { Multilevel } \\
\text { DA }\end{array}$ & $\begin{array}{l}\text { Multilevel } \\
\text { RA }\end{array}$ \\
\hline$R_{1}$ & $\mathrm{~m}$ & 2.38 & 2.032 & 2.034 \\
$R_{2}$ & $\mathrm{~m}$ & 3.38 & 3.218 & 3.475 \\
$h_{1} / 2$ & $\mathrm{~m}$ & 1.12 & 0.700 & 0.700 \\
$h_{2} / 2$ & $\mathrm{~m}$ & 0.37 & 0.241 & 0.463 \\
$d_{1}$ & $\mathrm{~m}$ & 0.19 & 0.332 & 0.331 \\
$d_{2}$ & $\mathrm{~m}$ & 0.65 & 0.400 & 0.178 \\
$J_{1}$ & $\mathrm{MA} / \mathrm{m}^{2}$ & 22.57 & 20.097 & 20.001 \\
$J_{2}$ & $\mathrm{MA} / \mathrm{m}^{2}$ & 11.06 & 19.998 & 19.993 \\
$B_{\text {stray }}$ & $\mathrm{mT}$ & 2.27 & 0.98 & 1.17 \\
$E$ & $\mathrm{MJ}$ & 178.75 & 179.63 & 180.22 \\
$B_{\text {max }}$ & $\mathrm{T}$ & $4.12(4.91)$ & $4.24(5.30)$ & $4.28(5.31)$ \\
FEM & --- & 4720 & 643 & 721 \\
$F$ & --- & 0.805 & 0.331 & 0.392 \\
\hline \hline
\end{tabular}

TABLE IV

SMES Performance AFter MCA of Multilevel Optimization

\begin{tabular}{llllll}
\hline \hline \multirow{2}{*}{ Par. } & \multirow{2}{*}{ Unit } & \multicolumn{2}{l}{ Deterministic } & \multicolumn{2}{l}{ Robust } \\
& & $\mu$ & $\sigma$ & $\mu$ & $\sigma$ \\
\hline$B_{\text {stray }}$ & $\mathrm{mT}$ & 1.00 & 0.034 & 1.19 & 0.017 \\
$E$ & $\mathrm{MJ}$ & 179.89 & 0.789 & 179.88 & 0.404 \\
\hline \hline
\end{tabular}

TABLE V

RELIABILITY (p) AND RoBUST LEVEL ( $\sigma$ ) OF THE SMES

\begin{tabular}{llcccc}
\hline \hline \multirow{2}{*}{ Par. } & \multicolumn{2}{c}{ Deterministic } & \multicolumn{2}{c}{ Robust } \\
& $\mathrm{p}$ & $\sigma$ & $\mathrm{p}$ & $\sigma$ \\
\hline$g_{1}$ & 0.9762 & 2.26 & 1 & $>6$ \\
$g_{2}$ & 1 & $>6$ & 1 & & $>6$ \\
POF & $2.38 \%$ & & $\approx 0.0 \%$ & \\
\hline \hline
\end{tabular}

(4) Table IV lists the MCA data $(\mu$ and $\sigma$ ) for SMES performance in terms of mean stray field and energy. Figs. 4 and 5 show their distributions for both DA and RA optimizations. As shown, the standard deviation of RA optimum is much smaller than that of DA, which means that the product quality (quality variation) of RA is better than DA.
(5) Fig. 6 shows the dimension comparison of SMES magnet after multilevel optimization under both DA and RA. As shown, the dimensions of the inner solenoid for both methods are almost identical, while the dimensions for the outer solenoid are obviously different. The gap between the windings given by DA is smaller than that of RA. Fig. 7 illustrates the filed distribution of the optimal RA optimum obtained from ANSYS. The illustration area is same as the one shown in Fig. 2. As shown, the maximal flux density in SMES is $4.27 \mathrm{~T}$, which is close to the optimized value $(4.28 \mathrm{~T})$, and both are much smaller than the limit $(5.31 \mathrm{~T})$, as shown in Table III).
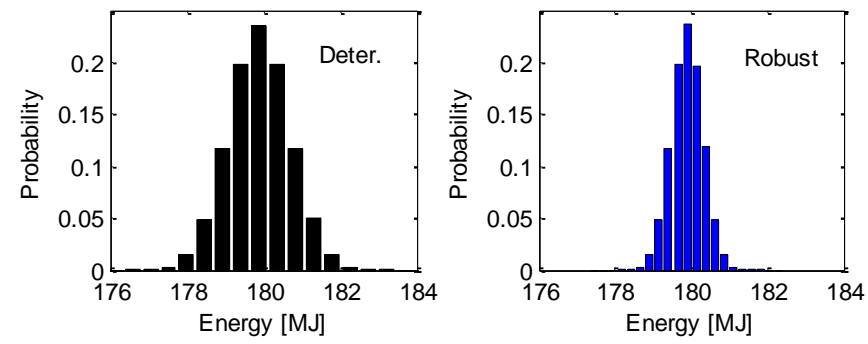

Fig. 4 Distributions of energy in SMES for both methods

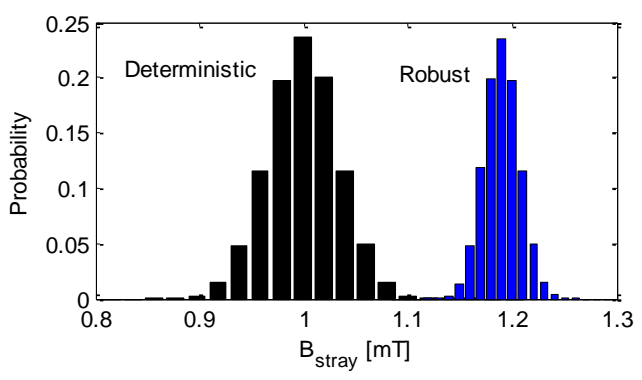

Fig. 5 Distributions of mean stray fields for both methods

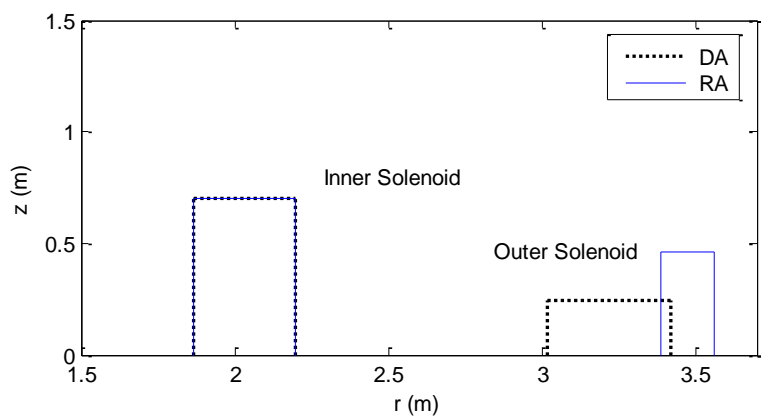

Fig. 6 Comparison of SMES magnet dimensions after optimization
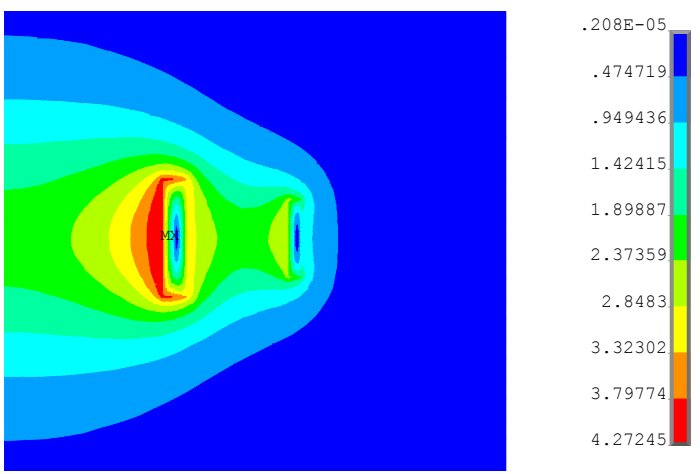

Fig. 7 Magnetic field distribution of the optimized SMES (axisymmetric) 


\section{CONCLUSIONS}

This work presented a multilevel optimization method for the robust design of a SMES based on a benchmark problem, TEAM problem 22. As shown, (1) the computation cost of FEM has been saved by $84.72 \%$; (2) the reliability of the designed SMES has been improved form $97.62 \%$ to $100 \%$ considering the manufacturing tolerance and operation variations of the coils; (3) product variation (standard deviation as shown in Figs. 4 \& 5) has been reduced greatly by taking the proposed method. Therefore, the proposed method benefits the industry applications of SMES.

Meanwhile, this method can be applied to design coils for other types of SMES, for example, HTS SMES. However, for HTS SMES, besides the electromagnetic analysis, mechanical analysis should be coupled as well. Then, besides the dimension and excitation current, other parameters, such as the winding turns and number of DPC mentioned in section II can be include in this optimization method too. Finally, for a given application with several different options in terms of coil topology and material, due to the high efficiency of this method, the designer is able to optimize each option and find out the best one (the global optimum) by comparison.

\section{REFERENCES}

[1] B.-K. Kang, S.-T. Kim, B.-C. Sung, and J.-W. Park, "A study on optimal sizing of superconducting magnetic energy storage in distribution power system," IEEE Trans. Appl. Supercond., vol. 22, no. 3, Article 5701004, 2012.

[2] P. Tixador, N. T. Nguyen, J. M. Rey, et al., "SMES optimization for high energy densities," IEEE Trans. Appl. Supercond., vol. 22, no. 3, Article 5700704, 2012.

[3] S. Ishiguri, and D. Kiuchi, "Performance improvement and optimization of new high-temperature superconducting coil assembly," IEEE Trans. Appl. Supercond., vol. 24, no. 2, Article 4901104, 2014.

[4] S. L. Lalitha, and R. C. Gupta, "The mechanical design optimization of a high field HTS solenoid," IEEE Trans. Appl. Supercond., vol. 25, no. 3, Article 4601504, 2015.

[5] A. H. Moghadasi, H. Heydari, and M. Farhadi, "Pareto optimality for the design of SMES solenoid coils verified by magnetic field analysis," IEEE Trans. Appl. Supercond., vol. 21, no. 1, pp. 13-20, 2011.

[6] J. K. Fang, J. Y. Wen, S. R. Wang, L. Ren, Y. J. Tang, X. T. Peng and Z. Chen, "Laboratory and field tests of movable conduction-cooled high temperature SMES for power system stability enhancement," IEEE Trans. Appl. Supercond., vol. 23, no. 4, Article 5701607, 2013.

[7] S. Kwak, M. Park, W. Kim, et al., "The optimal design of 600 kJ SMES magnet based on stress and magnetic field analysis," IEEE Trans. Appl. Supercond., vol. 18, no. 2, pp. 713-716, 2008.

[8] B. Vincent, P. Tixador, T. Lecrevisse, J.-M. Rey, X. Chaud, and Y. Miyoshi, "HTS magnets: opportunities and issues for SMES," IEEE Trans. Appl. Supercond., vol. 23, no. 3, Article 5700805, 2013.

[9] R. Gupta, M. Anerella, P. Joshi, J. Higgins, S. Lalitha, W. Sampson, J. Schmalzle, and P. Wanderer, "Design, construction, and testing of a large-aperture high-field HTS SMES coil," IEEE Trans. Appl. Supercond., vol. 26, no. 4, Article 5700208, 2016.

[10] L. Ren, W. P. Zuo, X. H. Shi, et al., "Development of a movable HTS SMES System", IEEE Trans. Appl. Supercond., vol. 25, no. 4, Article $5701109,2015$.

[11] P. Alotto, U. Baumgartner, F. Freschi, A. Köstinger, Ch. Magele, W. Renhart, and M. Repetto, "SMES optimization benchmark extended: introducing Pareto optimal solutions into TEAM22," IEEE Trans. Magn., vol. 44, no. 6, pp. 1066-1069, 2008.

[12] G. L. Soares, R. L. Adriano, C. A. Maia, L. Jaulin and J. A. Vasconcelos, "Robust multi-objective TEAM22 problem: a case study of uncertainties in design optimization," IEEE Trans. Magn., vol. 45, no. 3, pp.1028 -1031, 2009

[13] Y. H. Sung, D. W. Kim, and D. H. Kim, "Applying reliability assessment methods to superconducting magnetic energy storage system designs," IEEE Trans. Magn., vol. 47, no. 11, pp. 4623-4628, 2011.
[14] Z. Y. Ren, C. Park, and C. S. Koh, "Numerically efficient algorithm for reliability-based robust optimal design of TEAM problem 22," IEEE Trans. Magn., vol. 50, no. 2, Article \# 7016304, 2014.

[15] G. Lei, Y. G. Guo, J. G. Zhu, and K .R. Shao, "Sequential subspace optimization method for electromagnetic devices design with orthogonal design technique," IEEE Trans. Magn., vol. 48, no. 2, pp. 479-82, 2012.

[16] P. G. Alotto, U. Baumgartner, F. Freschi, et al., SMES optimization benchmark: TEAM workshop problem 22. 1996. [online] Available: online

[17] F. G. Guimarães, F. Campelo, R. R. Saldanha, et al. "A multiobjective proposal for the TEAM benchmark problem 22," IEEE Trans. Magn., vol. 42, no. 4, pp.1471-1474, .2006.

[18] F. Campelo, F. G. Guimarães, H. Igarashi, et al., "A clonal selection algorithm for optimization in electromagnetics," IEEE Trans. Magn., vol. 41 , no. 5, pp. 1736-1739, 2005

[19] G. Lei, J. G. Zhu, and Y. G. Guo., "Robust design optimization of PMSMC motors for Six Sigma quality manufacturing," IEEE Trans. Magn., vol. 49, no. 7, pp. 3953-3956, Jul. 2013.

[20] G. Lei, T. S. Wang, J. G. Zhu, and Y. G. Guo, "System level design optimization method for electrical drive system: robust approach," IEEE Trans. Ind. Electron., vol. 62, no. 8, pp.4702-4713, 2015.

[21] P. N. Koch, R. J. Yang, and L. Gu. "Design for six sigma through robust optimisation," Structural and Multidisciplinary Optimization, vol. 26, no. 3-4, pp. 235-248, 2004.

[22] X. J. Meng, S. H. Wang, J. Qiu, et al., "Robust Multilevel Optimization of PMSM Using Design for Six Sigma," IEEE Trans. Magn., vol. 47, no. 10 , pp. 3248-3251, 2011.

[23] G. Lei, T. S. Wang, J. G. Zhu, et al., "System level design optimization method for electrical drive system: deterministic approach," IEEE Trans. Ind. Electron., vol. 61, no. 12, pp. 6591-6602, 2014.

[24] G. Lei, Y. G. Guo, J. G. Zhu, et al., "Techniques for multilevel design optimization of permanent magnet motors", IEEE Trans. Energy Conver., vol. 30, no. 4, pp. 1574-1584, Nov. 2015. 